\title{
Evaluation Model of College Students' Entrepreneurship Success Rate Based on Grey Relational Theory
}

\author{
Xiaonan Yan $^{1}$, Jian Tian*1 \\ ${ }^{1}$ Jiangsu University Of Science And Technology, Jiangsu, China \\ ${ }^{*}$ Corresponding author: Jian Tian
}

\begin{abstract}
In order to improve the success rate of entrepreneurship of college students, it is necessary to establish a success rate evaluation model to analyze the success rate of entrepreneurship and make the optimal discrimination. However the current models failed to reach high accuracy due to the lack of factors set. To this end, this paper proposes a college students' entrepreneurial success evaluation model based on big data analysis, which is based on ambiguity comprehensive evaluation theory. Through building the evaluation factors set, calculating the relative impact of indicators, giving relative weights ratio of factors, and using data analysis. The experimental simulation results show that the model has high evaluation accuracy, and therefore has practical significance in improving the success rate of college students' entrepreneurship.
\end{abstract}

\section{Introduction}

In recent years, the entrepreneurship enthusiasm of college students is increasing drastically. The Ministry of Education pointed out that college graduates scaled up to 8.34 million in 2019 and the entrepreneurship-driveemployment mode becomes the new solution. Although the entrepreneurial enthusiasm continues to grow, the rate of entrepreneurial failure remains high due to defective supporting system, hiatus of entrepreneurship education, and lack of successful business profit mode. Therefore, it is crucial to establish an entrepreneurship success rate evaluation model to break through the dilemma and researchers have attached great importance on developing an accurate evaluation model.[1]

With the advent of the era of AIOT, big data has gradually become the most important means to solve the problem of college students' success rate evaluation. McKinsey defines big data as large set of data can be captured, stored, managed and analyzed beyond the capabilities of conventional database software tools. Big data gradually is characterized by large scale, diverse information types and fast transmission speed, which poses a technical challenge to designing the college students' entrepreneurship success rate evaluation model.

At present, many scholars have conducted a large number of studies on the evaluation model of college students' entrepreneurial success rate. Fu Xueqian, Chen Haoyong and others constructed a comprehensive evaluation system for the success rate of college students' entrepreneurship, obtained the optimal evaluation strategy for the success rate, and established an evaluation model for the success rate of college students' entrepreneurship. This method can quickly search out the main factors that lead to entrepreneurial risk, but failed to assign the weight of the index towards success. Tian Shiming, Pan Mingming used similar trapezoidal fuzzy number method to establish the mapping relationship between business and market risk, which made fuzzy number accurate by expectations functions. This method can calculate the value of each success rate index.[2] However, it is unable to construct the factor set of the success rate of college students, and the evaluation accuracy is low. $\mathrm{Xi}$ Rongrong and Yun Xiaochun defined the concept of success rate of college students' entrepreneurship based on statistical characteristics, obtained the observation sequence of success rate, and established the success rate evaluation model to evaluate the success rate of college students' entrepreneurship. This method can calculate the proportion of college student's entrepreneurial success characteristic value, but it cannot extract the characteristics that affect the success rate index.[3]

In view of the above problems, this paper proposes an evaluation model of success rate of college students' entrepreneurship based on big data analysis. By constructing the evaluation factor set of success rate of college students' entrepreneurship, the overall success rate of college students' entrepreneurship is determined based on this. Finally, through experimental simulation, the optimization of the success rate of college students' entrepreneurship is determined.[4]

\section{Modeling principle}

To design an entrepreneurship success rate evaluation model, the specific steps are as follows:

Assuming that $s^{\prime}$ represents the entrepreneurial event of college students to be evaluated, $\left\{d^{\prime}, b^{\prime}, m^{\prime}, k^{\prime}\right\}$ represents the four indicators of the evaluation system, 
$v_{d f g}^{\prime}$ represents the value of each indicator, and $R_{\text {hhurer }}^{\prime}$ represents the core competitiveness, formula (1) is used to establish the evaluation indicator system:

$$
Q_{\text {sef }}^{\prime}=\frac{R_{\text {hhwer }}^{\prime} \mp v_{d f g}^{\prime}}{\left\{d^{\prime}, b^{\prime}, m^{\prime}, k^{\prime}\right\}} \oplus \frac{d_{s d g}^{\prime}}{s^{\prime}} \oplus g_{g h j}^{\prime}
$$

In the above formula, ${ }^{d}{ }^{\prime}{ }^{\prime}$ g represents the type of evaluation index for college students' entrepreneurial success rate, and $g^{\prime}{ }^{\prime h j}$ represents the level of college students' entrepreneurial core competitiveness. [5]

It is assumed that $e^{\prime \prime}{ }_{e r t}$ represents the specific value of $R_{i}^{\prime}, \quad \eta_{\text {tion }}^{\prime}$ represents the potential of entrepreneurial competitiveness, and $q_{d g j}^{\prime}$ represents the set of entrepreneurial success factors. Formula (2) is used to grade the indicators that affect the entrepreneurial success of college students:[5]

$$
v_{\text {swerk }}^{\prime}=\frac{e^{\prime \prime}{ }_{e r t} \mp R_{i}^{\prime}}{\eta_{k i o u}^{\prime}} \times \frac{q_{d g j}^{\prime} \oplus z_{s f y}^{\prime}}{d_{r t p}^{\prime} \times k_{j}}
$$

In the above formula, $z_{s f y}^{\prime}$ represents the clustering threshold of the evaluation index for college students. ${ }^{\prime}{ }_{n p}$ represents the clustering weight of the decision index $k_{j}$.

Assuming that $\beta_{d f^{\prime}}^{\prime}$ represents the decision coefficient of the decision object $o^{\prime}$ under the grey category of ${ }^{k_{d f g}^{\prime}}$, $\varepsilon_{\text {frty }}^{\prime}$ represents the decision coefficient vector of the decision object $o^{\prime}$, and $W^{\prime}$ represents the decision coefficient matrix of the entrepreneurial success rate, then formula (3) is used to express the importance of each success rate evaluation factor:[6]

$$
A_{s w e r}^{\prime}=\frac{W_{a q u e}^{\prime} \times \beta_{d f}^{\prime}}{o^{\prime} \pm p_{f g}^{\prime} \times \varepsilon_{f r t y}^{\prime}} \times k_{d f g}^{\prime}
$$

$p_{\text {fs }}^{\prime}$ represents the frequency.

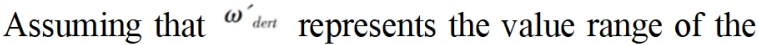
secondary influence index in the index system and $\mu_{f r t}^{\prime}$ represents the clustering weight of index, then formula (4) is used to establish the success rate judgment matrix:

$$
a_{\text {sdfhk }}^{\prime}=\frac{\mu_{f r t}^{\prime} \times \omega_{d e r t}^{\prime}}{A_{\text {swer }}^{\prime} \times v_{\text {swerk }}^{\prime}} \oplus e_{d f g h}^{\prime}
$$

$e^{\prime}{ }_{d j g h}$ represents the sample matrix of the evaluation.

Assuming that $\boldsymbol{\sigma}^{\prime}$ wert represents the number of sample types for success rate evaluation, ${ }^{\prime}{ }_{\text {nerf }}$ represents the sample type characteristics for success rate evaluation, and $x^{\prime \prime}$ sdfgh represents the indicator level vector for success rate evaluation, then equation (5) can be used to obtain the comprehensive correlation degree of college students' entrepreneurial success potential level:

$$
A_{\text {qwert }}^{\prime}=\frac{x^{\prime \prime}{ }_{\text {sdfgh }} \times k_{\text {wervf }}^{\prime}}{\boldsymbol{\sigma}^{\prime}{ }_{\text {wert }}} \mp f^{\prime^{\prime}{ }_{d f g h}}
$$

$f^{\prime \prime}$ "dfgh represents the interaction between indicators.

Assuming that ${ }^{d}$ sumbly $^{\prime}$ represents the state of entrepreneurship and $q_{\text {sdfg }}^{\prime}$ represents the probability of success rate, formula (6) is used to establish the success rate evaluation model:

$$
w_{\text {sddf }}^{\prime}=\frac{q_{\text {sdfg }}^{\prime} \times d_{\text {sdfhhg }}^{\prime}}{A_{\text {qwert }}^{\prime}} \times \frac{a_{\text {sdfhk }}^{\prime} \times A_{\text {swer }}^{\prime}}{v_{\text {swerk }}^{\prime}} \oplus Q_{\text {sef }}^{\prime}
$$

Above is the modeling principle where the evaluation model is based.

\section{Establishment of the model}

\subsection{Weight calculation of evaluation factors}

The accuracy of other evaluation model currently is insufficient. It is necessary to use the fuzzy comprehensive evaluation theory to construct the evaluation factor set, calculate the weight, obtain the relative ratio, and then establish the evaluation matrix for the success rate factors. The specific steps are as follows:[6]

Define all relative factors as evaluation factors set and assume $i_{j i}, j^{\prime} \quad$ representing the ordinal number of the first and $j_{j i}$ secondary factors, $d^{\prime \prime}{ }_{\text {wer }}$ representative factors hierarchies of threshold, $\gamma_{s d f^{\prime}}^{\prime}$ the college students' entrepreneurial market risk, the use of type (7) build the evaluation of college students' entrepreneurial success factors set:

$$
s_{d f g h}^{\prime}=\frac{\gamma_{s d f}^{\prime} \times j_{j i}^{\prime}}{i_{j i}^{\prime} \mp d^{\prime \prime}{ }_{w e r}} \mp y_{g t y u}^{\prime}
$$

$y^{\prime}{ }_{g t y u^{\prime}}$ stands for leader quality.

Assuming that $s_{s f g h}^{\prime}$ represents the stability of the team, $w^{\prime}{ }^{\prime}$ rh represents the success rate of HRM, ${ }^{l^{\prime}{ }_{k j}^{\prime}}$ represents the conflict between entrepreneurship and study, and $Q^{\prime}$ swen represents the risk of insufficient initial capital, the weight of each factor is calculated by using Equation (8) :

$$
k_{j k l l}^{\prime}=\frac{Q_{\text {swert }}^{\prime} \times s_{s f g h}^{\prime}}{w_{e r h}^{\prime}} \times l_{k j}^{\prime}
$$

Assuming that $l^{\prime}{ }_{h j k}$ represents the success rate of capital operation turnover and $\boldsymbol{\sigma}^{\prime}$ sdfg $^{\prime}$ represents the success rate of enterprise operation and management, equation (9) is used to obtain the relative ratio of weight of each factor:

$$
b_{f g h j}^{\prime}=\frac{\boldsymbol{\varpi}_{s d f g}^{\prime}}{v_{d f g h j}^{\prime} \mp w_{d f g}^{\prime}} \times l_{h j k}^{\prime}
$$

$v^{\prime}{ }_{d f g h j}$ represents the index weight column vector, and $w_{d f g}^{\prime}$ represents the consistency of evaluation criteria.

Assuming that $d^{\prime}{ }_{d f g f}$ represents the tacit knowledge of each expert, ${ }^{\omega_{d f g h}}$ represents the structure multiplied by the weight vector of the index, and $f^{\prime} d f^{\prime}$ th represents the membership degree of the evaluation level, then the evaluation matrix can be established by using Equation (10) :

$$
\begin{gathered}
F_{\text {swen }}^{\prime}=\frac{f_{d f g h}^{\prime} \times d_{d f g f}^{\prime}}{\omega_{d f g h}^{\prime}} \oplus \frac{j_{d g h}^{\prime} \times s_{d f g h}^{\prime}}{b_{f g h j}^{\prime}} \pm k_{j k l l}^{\prime} \\
j_{d g h h}^{\prime} \text { represents the fuzzy weight vector. }
\end{gathered}
$$




\subsection{An optimized evaluation model based on data analysis}

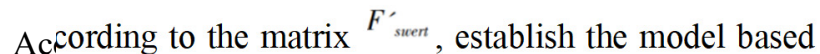
on the data analysis. The specific steps are as follows:

It is assumed that $\partial_{s f g}^{\prime}$ represents the weight of the first-level index to the target layer, ${ }^{\mu_{d g g h}^{\prime}}$ represents the maximum eigenvalue of the success rate, and $f_{f g h j}^{\prime}$ represents the consistency test result. Based on the success factor evaluation matrix $F^{\prime}$ sect , formula (11) is used to assign the weight of the index based on data analysis:

$$
A_{\text {wert }}^{\prime}=\frac{\partial_{s f g}^{\prime} \mp \mu_{d g g h}^{\prime}}{f_{f g h j}^{\prime} \times F_{\text {swen }}^{\prime}} \mp m_{h j k l}^{\prime} \mp g^{\prime}{ }_{h j k}
$$

$m^{\prime}{ }_{h j k l}$ represents the weight value of the first-level index, and ${ }^{g^{\prime}{ }_{j \cdot k}^{\prime}}$ represents the evaluation index score by experts.

Assuming that $\lambda_{\text {imerr }}$ represents scores given by expert $k_{s f f}^{\prime}, p_{f g}^{\prime} \quad$ represents the ordinal number of the gray category, and $E_{\text {stuen }}^{\prime}$ represents the whitenization weight function, then formula (12) is used to determine the gray category:

$$
q_{d j p}^{\prime}=\frac{E_{s \text { seren }}^{\prime} \times \lambda_{\text {bmuer }}^{\prime}}{k_{s f f}^{\prime} \mp p_{f g}^{\prime}} \pm \iota_{d g}^{\prime}
$$

$\iota_{d g}^{\prime}$ represents the vector of grey evaluation coefficient.

Assuming that $\mu_{\text {aurt }}^{\prime}$ represents the grey evaluation weight vector, the it is calculated by Formula (13) :

$$
A_{\text {suef }}^{\prime}=\frac{\mu_{a u r t}^{\prime} \times q_{d j p}^{\prime}}{r_{s f h j}^{\prime}} \mp m_{d g j}^{\prime}
$$

$q_{d j p}^{\prime}$ represents the grey evaluation weight, $\eta^{\prime}{ }_{d g h}$ represents any secondary index, and $m_{d g j}^{\prime}$ represents the set of grey evaluation weight vectors.

Assuming that ${ }^{d_{d f \text { po }}}$ represents the average annual income growth rate of the start-up and $\eta_{\text {dgh }}^{\prime}$ represents the number of introduced talents, then formula (14) can be used to obtain each grey evaluation coefficient:

$$
f^{\prime \prime}{ }_{\text {stuer }}=\frac{\eta_{d g h}^{\prime} \times r_{s f h j}^{\prime}}{\left\{X_{\text {ojg }}^{\prime}\right\} \times\left\{X_{j g}\right\}} \mp \frac{y u_{i}^{\prime} \times u_{i}^{\prime}}{d_{d f p o}^{\prime}} \oplus p_{l p o}^{\prime}
$$

$\left\{X_{o j k}\right\},\left\{X_{i k}\right\}$ respectively represents the grey evaluation weight of all evaluators, and $p^{\prime}{ }_{\text {roo }}$ represents the grey evaluation weight vector of the secondary index $r^{\prime}{ }_{s / j}$.

Assuming that $\sigma^{\prime}$ in re represents the grey evaluation weight matrix of ${ }^{r_{s f j i}}, \mu^{\prime}{ }_{\text {ent }}$ represents the weight vector of the total index, and ' $\eta_{\text {sef }}^{\prime}$ represents the vector formed by the assignment of each grey class according to grey level, formula (15) is used for the comprehensive evaluation of the indicators:

$$
Q_{\text {sedg }}^{\prime \prime}=\frac{\eta_{\text {swef }}^{\prime} \mp \mu_{e r t}^{\prime}}{\varpi_{j \text { jert }}^{\prime}} \mp r_{\text {sfhj }}^{\prime}
$$

Assuming that $d_{d f}^{\prime}$ representing the maximum value of the weight of the evaluation index, formula (16) is used to establish an optimal evaluation model for the success rate of college students' entrepreneurship:

$$
d_{g y h}^{\prime}=\frac{d_{d f}^{\prime} \mp A_{\text {wert }}^{\prime}}{A_{\text {seef }}^{\prime} \times Q^{\prime \prime} \text { sedg }^{\prime}} \times q^{\prime}{ }_{d j p} \mp f^{\prime \prime}{ }_{\text {ster }}
$$

\section{Experimental and simulatian proof}

Aiming at the success rate evaluation $n_{10 d e l}$, a platform was built under the simulation $e_{n v i r o n m e n t}$ for experiments. The experimental data are $\mathrm{fr}_{\mathrm{om}}$ the sales data set of the entrepreneurship park of a university from 2016 to 2019 .

\subsection{Setting of evaluation indexes}

In the experiment, in order to better verify the feasibility of the success rate evaluation of college students based on the big data analysis model, the experiment was divided into two stages. In the first stage, the consistency of the evaluation was used as the evaluation index to define the accuracy of the success rate evaluation of college students based on the big data analysis model. In the second stage of the experiment, in order to highlight the comprehensiveness and fairness of the experiment, the statistical model proposed in the literature was used as a comparison model for common analysis and comparison, and the quality of different models for the success rate evaluation of college students' entrepreneurship was defined from the aspects of the comprehensiveness of the evaluation and the compliance rate of the evaluation.[8]

(1) The consistency of the evaluation is defined as the degree of fitting between the entrepreneurial success rate of the evaluation and the actual results. Suppose that ${ }^{d_{d j g}}$ represents income (net profit), ${ }^{\prime}$ ste represents pre-tax profit, and $e_{f g}^{\prime}$ represents annual profit. Formula (17) is used to calculate the consistency of evaluation ${ }^{l^{\prime}}$ sedg :

$$
l_{\text {sedg }}^{\prime}=\frac{d_{d f g}^{\prime}}{o_{s w e}^{\prime}} \mp e_{f g}^{\prime}
$$

It can be seen from the calculation results of Equation (17) that the higher the $l_{\text {sxdg }}^{\prime}$ value is, the higher the evaluation accuracy will be.

(2) The comprehensiveness of the evaluation is defined as the comprehensiveness of the established evaluation mechanism for college students' entrepreneurial success rate. Then, equation (18) is used to calculate the comprehensiveness of the evaluation ${ }^{\prime} d / f$ :

$$
s_{d f g}^{\prime}=l_{s e d g}^{\prime} \mp \frac{d_{d f g}^{\prime}}{o_{s w e}^{\prime}} \mp e_{f g}^{\prime}
$$

(3) The assessed compliance rate is defined as the difference between the assessed success rate of college students in starting a business and the actual success rate of college students in starting a business. Use Equation (19) to calculate the assessed compliance rate $\mu_{j u i}^{\prime}$ :

$$
\mu_{j u}^{\prime}=s_{d f g}^{\prime} \mp l_{s e d g}^{\prime} \mp \frac{d_{d f g}^{\prime}}{o_{s w e}^{\prime}} \mp e_{f g}^{\prime}
$$


The calculation results of Equations (18) and (19) show that the higher the ${ }^{s_{d / f}^{\prime} / \mu_{j u}^{\prime}}$ value is, the higher the evaluation quality will be.[9]

\subsection{Consistency test of the model}

The test results were shown in Figure 1.

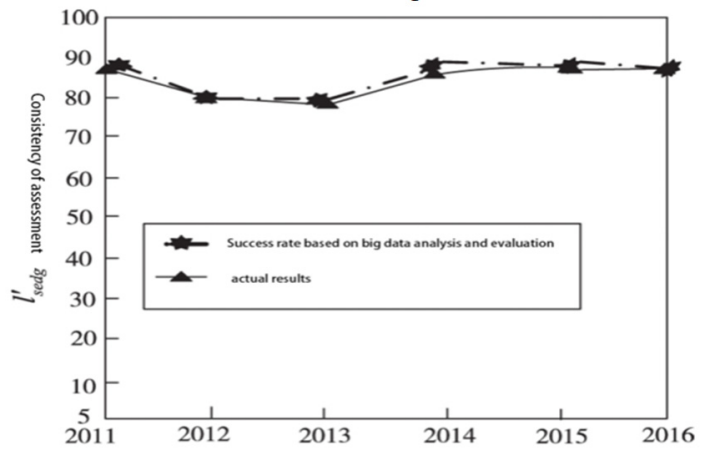

Fig1. Consistency of the model

It can be concluded from the experimental simulation results in Figure 1 that the model can directly reflect the success rate of college students' entrepreneurship, which has important practical guiding significance.[10]

\subsection{Evaluation by different models}

The big data analysis model were used to carry out the success rate evaluation experiment of college students' entrepreneurship, and the comprehensiveness and compliance rates of different models were compared. The comparison results were shown in Figure 2 and Figure 3.

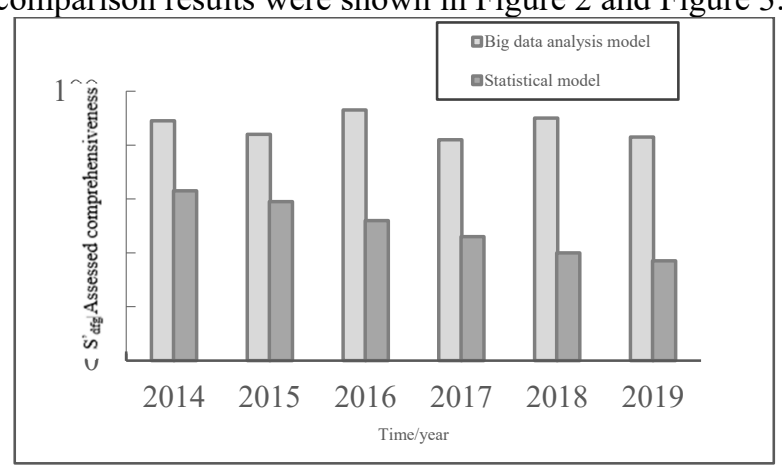

Fig2. Comprehensiveness of different models

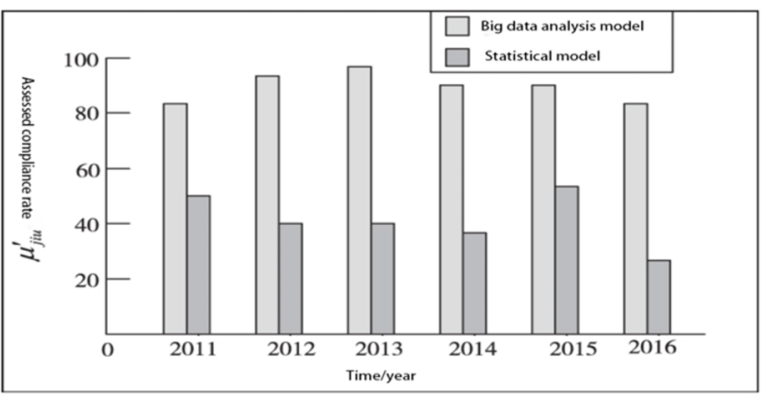

Fig3. Compliance rates of different models

From figure 2 and 3 can be analyzed that our model can guarantee evaluation quality more and the simulation results show that the model has high accuracy and effectively improves the success rate of students' entrepreneurship.[11]

\section{Conclusion}

Aiming at college students' success rate evaluation, this paper proposes an evaluation model based on big data analysis. This model constructs the evaluation factor set, determines the overall success rate, and realizes optimal evaluation of the success rate. The experimental simulation proves that, compared with previous evaluation model, the evaluation accuracy of this paper is relatively high, which enables students to effectively evaluate their entrepreneurship and improves success rate of college students' entrepreneurship.[12]

\section{Reference}

1. Ames, Manyuka et al. Big Data: The Next Frontier for Innovation, Competition and Productivity[J] Las Vegas: The McKinsey Global Institute, 2014.

2. Victor Mike Schonberger, Kenneth Cukier. The Age of Big Data: The Revolution of life, Work and Thinking $[\mathrm{M}]$, translated by Sheng Yangyan and Zhou Tao. Hangzhou: Zhejiang People's Publishing House, 2012:13.

3. Liu Shiwei. Algorithm Design of Entrepreneurial Risk Evaluation for College Students Based on Big Data Analysis [J]. Modern Electronic Technology, 2018:125-128.

4. Tang Xinyu, Chen Xiaoming. Algorithm Design and Research of Deep Computing Evaluation Model Based on Big Data Characteristics [J]. Software Engineering, 2018: 39-41.

5. Lv M. Design of sports Competition Evaluation System Based on Big data and Motion Recognition Algorithm [J]. Electronic Design Engineering, 2019:6-10.

6. J.S Vockler, Deelman et al. Experiences using cloud computing for a scientific application. Proceedings of the 2 nd international workshop on scientific cloud computing, 2011.

7. C. O 'Neil, R. Schutt. Doing Data Science for Business. O 'Reilly Media, First Edition, 2013(7).

8. Zheng Shiming. Innovation and Entrepreneurship Education Reform driven by Big Data: Theory and Practice [J], Tsinghua University Education Research, 2016(05):65-73.

9. $\mathrm{Fu}$ Xueqian, Chen Haoyong, Liu Guote et al. Comprehensive power quality Evaluation Method for distributed power Supply [J]. Chinese journal of electrical engineering, 2014,34 (25):4270-4276.

10. Tian Shiming, Pan Mingming, Yuan Jiahang, et al. Research on power supply risk Evaluation method for Active and Important Users [J]. Power grid technology, 2015:35-41.

11. Xi Rongrong, Yun Xiaochun et al. An improved quantitative evaluation method for network security situation [J]. Journal of computer science, 2015:749758. 